\title{
Prophylaxis Pre-Operative Single Dose Antibiotic Versus Conventional Antibiotic Therapy in Lichtenstein Tension Free Mesh Repair
}

\author{
K. Saravanan ${ }^{1}$, A. Muthukumar ${ }^{2}$ \\ ${ }^{1}$ Associate Professor, Department of Surgery, Government Theni Medical College, Tamilnadu, ${ }^{2}$ Assistant Professor, \\ Department of Surgery, Government Theni Medical College, Tamilnadu, India
}

Corresponding author: Dr. A. Muthukumar, Assistant Professor, Department of Surgery, Government Theni Medical College, Tamilnadu, India

DOI: http://dx.doi.org/10.21276/ijcmsr.2019.4.2.37

How to cite this article: K. Saravanan, A. Muthukumar. Prophylaxis pre-operative single dose antibiotic versus conventional antibiotic therapy in lichtenstein tension free mesh repair. International Journal of Contemporary Medicine Surgery and Radiology. 2019;4(2):B170-B172.

\section{A B S T R A C T}

Introduction: Surgical antibiotic prophylaxis (SAP) refers to the utilization of antibiotics for the prevention of surgical site infections (SSIS). SSIs impact on both the patient and health care system through increased mortality and morbidity, prolonged hospital admission, reduced the quality of life and additional financial costs for ongoing inpatient and outpatient treatment. Aim: To study the effect of single-dose antibiotic prophylaxis given 30 minutes prior to surgery with conventional antibiotic therapy.

Material and Methods: Total of 50 patients were randomized to 25 each group. All patients in the study group undergoing surgery were given $400 \mathrm{mg}$ parenteral ciprofloxacin $30 \mathrm{~min}$ prior to surgery. In the control group, the patients were given 2 days parenteral ciprofloxacin $400 \mathrm{mg}$ twice a day and the next 5 days the same antibiotics were given in oral route, after surgery. The outcome in terms of duration of surgery, surgical site infection, cost and antibiotic side effects were then compared.

Results: Out of 50 patients, the incidence of postoperative infection 10\% (5 patients), 3 cases in the control group and 2 patients prophylactic antibiotic group. No change in the management protocol was done. None developed antibiotic side effects in the study group. Control group patients developed study effects of nausea, vomiting and diarrhoea.

Conclusion: Prophylactic single-dose antibiotic is effective in preventing surgical site infection and is cost-effective in patients undergoing Lichtenstein tension-free mesh repair.

Keywords: Antibiotics, Conventional Therapy, Cost-effective, MESH Repair, Prophylaxis, Surgical Site Infection

\section{INTRODUCTION}

Antibiotics play a particularly important role in the postoperative care of patients undergoing elective surgery because of the high incidence of surgical site infection (SSI). It has been reported that $30-40 \%$ of patients experience post-operative SSI when a prophylactic antibiotic is not administered. ${ }^{1,2}$ Although several recent studies have indicated that single-dose (SD) administration may be as effective as multiple-dose, ${ }^{3}$ controversy still exists concerning the frequency and appropriate combination of antibiotics. In adults of all ages, SSIs represent a significant financial burden and are associated with increased length of hospitalization, re-admission, and mortality. It has been reported that patients who develop SSIs have a mortality rate that is $2-11$ times higher than that of patients who do not develop SSIs, and the mortality rate for SSIs is up to $6 \% .{ }^{4,5}$ Preventative measures in the preoperative period have changed rapidly over the past few decades. A large volume of research has established the importance of a host of preventative measures in the operative period. Examples include skin decontamination, perioperative warming, and antimicrobial prophylaxis. ${ }^{6,7}$ Surgical antibiotic prophylaxis given at induction of anesthesia is recommended for any surgery apart from clean procedures not involving an implant or prosthesis. Antibiotics should be specific and targeted to the likely causative organisms and appropriate for the patient taking account of allergies and comorbidities. The administration of the dose should occur no earlier than 120 minutes prior to the incision being made (WHO). Prolongation of prophylactic antibiotics after the operation is not recommended in the prevention of SSI. ${ }^{8}$

Current study aimed to investigate the effect of single-dose antibiotic prophylaxis given 30 minutes prior to surgery with conventional antibiotic therapy.

\section{MATERIAL AND METHODS}

This prospective comparative study was conducted department of General Surgery, Government Theni Medical College Hospital in elective Lichtenstein tension-free mesh 
repair for inguinal hernia. Patients were selected randomly as per inclusion and exclusion criteria and divided into 2 groups. Both gender more than 18 years were considered. Patient informed consent was obtained before surgery.

Study group: One dose of parenteral Ciprofloxacin 400mg IV after test dose $30 \mathrm{~min}$ prior to surgery and no more antibiotics were prescribed.

Control group: No pre-operative antibiotic given. In the post-operative ward for the first 2 days, IV antibiotics were given as follows:

- Parenteral ciprofloxacin 400mg IV bd.

- Next 5 days: tablet ciprofloxacin $500 \mathrm{mg}$ oral bd.

All the patients posted for these elective surgeries were admitted on the day prior to surgery. All necessary investigations were done and anaesthetic fitness obtained. The operative site was cleaned/shaved with aseptic precaution. All patients were asked to take body wash with soap on the day of surgery and the operative site was covered with a sterile dressing.

Asepsis is maintained, and checklists were verified. Standard surgical scrub for 5-10 min was mandatorily followed by the surgical team. Asepsis is maintained, and checklists were verified. Standard surgical scrub for 5-10 min was mandatorily followed by the surgical team. Southampton scoring system was used to grade the infection.

\section{RESULTS}

Total of 50 patients undergoing Lichtenstein tension-free mesh repair for inguinal hernia was divided into two groups. Patients in the control group were given, 7 days of antibiotics. Study group patients got only one dose of prophylactic

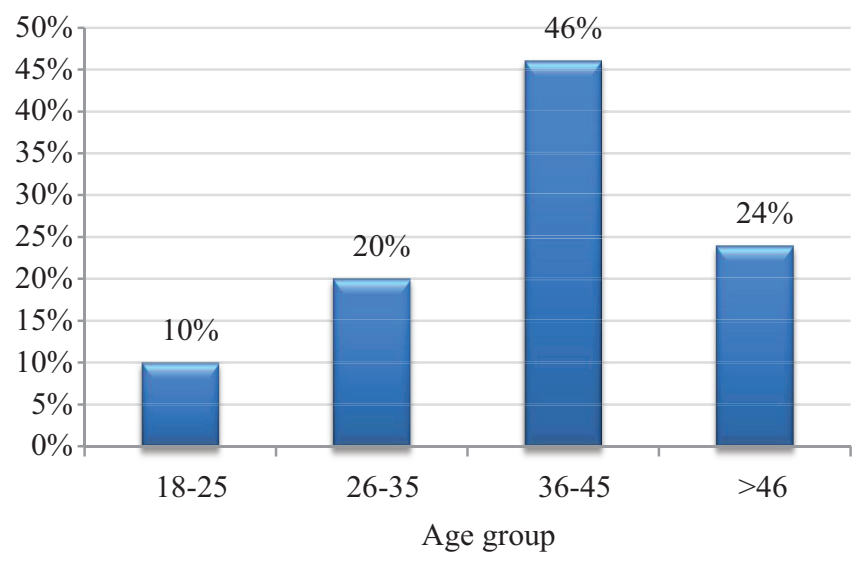

Figure-1: Distribution of Age group

3

- Study group —Control group

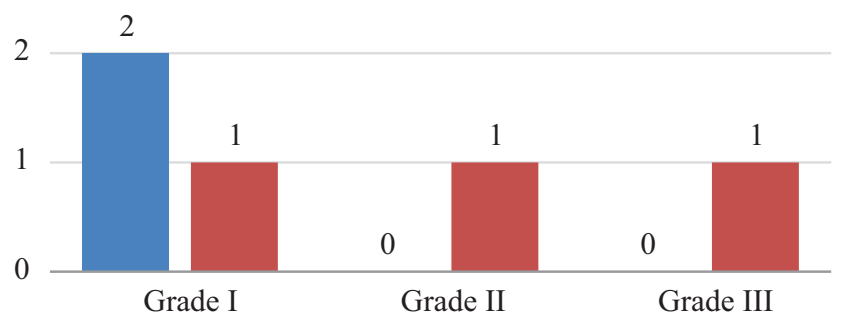

Figure-2: Grade of post-operative infections. antibiotic 30 minute before surgery.

In this study, the study group consists of 16 male and 4 female and in control group 12 male and 13 female patients were included. Most of the patients were in between 20 to 40 years in both groups. There was no statistical difference noted in weight, hemoglobin and duration of surgery. Both groups underwent spinal anesthesia (Figure-1).

Out of 50 patients, the incidence of postoperative infection $10 \%$ (5 patients), 3 cases in the control group and 2 patients prophylactic antibiotic group. No change in the management protocol was done. On appropriate local wound management, infections were controlled. No statistically significant difference with respect to infection profile was noted in both the groups. None developed antibiotic side effects in the study group. Control group patients developed study effects of nausea, vomiting and diarrhea (Figure-2).

\section{DISCUSSION}

The benefit of antimicrobial prophylaxis was reported as far back as the 1960s from randomized trials, and this practice has had a marked impact on surgical practice. General guidelines with respect to prophylaxis advocate the necessity for high tissue concentrations of antibiotics at the time when bacterial contamination is most likely to occur, i.e., from the first incision. Typically, prophylactic antibiotics are administered intravenously at the induction of anesthesia. A seminal study of 7000 patients confirmed there is variation in clinical practice with regards to the timing of prophylaxis. Administration of prophylactic antibiotics two hours prior to surgery is effective in reducing SSI. The administration of a first dose earlier than this prior to surgery or post-operatively is ineffective as a prophylactic measure. ${ }^{9-11}$

Borade $\mathrm{S}$ et al. reported 3\% of superficial surgical site infection in his study. ${ }^{12}$ Jogdand S et al. studied 183 clean surgical procedure with single dose antibiotics and found that multiple-dose antibiotics requirement after surgery was reduced. ${ }^{13}$ Shah YD et al. compared single dose antibiotics prior to surgery with multiple dose antibiotics treatment, and reported $11 \%$ of SSI in single dose regimen and multiple dose antibiotic regimen in patients undergoing laparoscopic surgery and reported that a single dose of antibiotics are more patient compliant, cost-effective, less adverse effects and prevents the emergence of antibiotic resistance. ${ }^{14}$

Most studies have reported an incidence of wound infection of $2.7-11.3 \%$ in patients who underwent open colectomy compared with 5.7-26\% in laparoscopic colectomy. ${ }^{15-17} \mathrm{~A}$ meta-analysis of post-operative wound infection reported a significantly lower incidence of SSI after laparoscopic colectomy $(3.9 \%, 21 / 537)$ than after open colectomy $(8.3 \%$, $43 / 518 ; P=0.005)$. In the present study, the rates of SSI in open and laparoscopic surgery were $16.6 \%(4 / 24)$ and $8.7 \%$ (6/69), respectively $(P=0.278)$. It seems to show that skillful surgical technique is more important than wound length for preventing wound contamination. ${ }^{18}$

\section{CONCLUSION}

Antibiotic prophylaxis is widely accepted as a means of decreasing postoperative infectious morbidity and is recommended for surgery. Single dose prophylactic antibiotic 
will be effective in reducing postoperative infection proper aseptic precautions during surgery, proper sterilization procedure of the operation theatre, correction of anemia should be done before surgery, not after, so that tissue can carry more oxygen and take the benefit of prophylactic antibiotic.

\section{REFERENCES}

1. Jeong WK, Park JW, Lim SB, Choi HS, Jeong SY. Cefotetan versus conventional triple antibiotic prophylaxis in elective colorectal cancer surgery. J. Korean Med. Sci. 2010; 25(2): 429-34.

2. Platell C, Hall JC. The prevention of wound infection in patients undergoing colorectal surgery. J. Hosp. Infect. 2001; 49(4): 233-8.

3. Fujita S, Saito N, Yamada $\mathrm{T}$ et al. Randomized, multicenter trial of antibiotic prophylaxis in elective colorectal surgery. Arch. Surg. 2007; 142(6): 657-61.

4. Korol E, Johnston K, Waser N, Sifakis F, Jafri HS, Lo M, Kyaw MH. A systematic review of risk factors associated with surgical site infections among surgical patients. PLoS One. 2013;8(12):e83743.

5. Teillant A,GandraS,BarterD,MorganDJ,Laxminarayan $R$. Potential burden of antibiotic resistance on surgery and cancer chemotherapy antibiotic prophylaxis in the USA: a literature review and modelling study. Lancet Infect Dis. 2015;15(12):1429-37.

6. Mangram AJ, Horan TC, Pearson ML, et al. Guideline for prevention of surgical site infection, 1999. Hospital Infection Control Practices Advisory Committee. Infect Control Hosp Epidemiol 1999;20(4):250-78 [quiz: 279-80].

7. Alexander JW, Solomkin JS, Edwards MJ. Updated recommendations for control of surgical site infections. Ann Surg 2011;253(6):1082-93.

8. Surgical site infections: prevention and treatment. Updated February 2017. NICE, https://www.nice.org. uk/guidance/CG74/ chapter/1-Guidance (accessed 29 Dec 2018).

9. Hill C, Flamant R, Mazas F et al. Prophylactic cefazolin versus placebo in total hip replacement: report of a multicentre double blind randomised trial. Lancet 1981; 11;1(8224): 795-96

10. Classen D, Evans RS, Pestotnik SL et al. The timing of prophylactic administration of antibiotics and the risk of surgical wound infections. N Engl J Med 1992; 326 (5)281-86

11. Burje JF. The effective period of preventive antibiotic action in experimental incision and dermal lesions. Surgery 1961;50(1):161-68

12. Borade S, Syed O. Single dose antibiotic prophylaxis for prevention of surgical site infection in elective surgery. International Surgery Journal. 2017;5(1):27.

13. Jogdand S, Shinde R, Chandrakar N. Outcome of Evidence-based Allocation of Single-dose Antibiotic extended to Three-dose Antibiotic Prophylaxis in Surgical Site Infection. International Journal of Recent Surgical and Medical Sciences. 2017;3(2):79-84.

14. Shah YD, Thekdi PI, Raut S, Patel KG. Single shot versus multiple shot antibiotic therapy in patients undergoing laparoscopic surgery: our experience. Int J
Res Med Sci 2013;1(5):252-6.

15. PoonJT,LawWL,Wong IW et al.Impact of laparoscopic colorectal resection on surgical site infection. Ann. Surg. 2009; 249(8): 77-81.

16. Romy S, Eisenring MC, Bettschart V, Petignat C, Francioli P, Troillet N. Laparoscope use and surgical site infections in digestive surgery. Ann.Surg. 2008; 247(3): 627-32.

17. Nakamura T, Mitomi H, Ihara A et al. Risk factors for wound infection after surgery for colorectal cancer. World J. Surg. 2008; 32(1): 1138-41.

18. Abraham NS, Young JM, Solomon MJ et al. Metaanalysis of short-term outcomes after laparoscopic resection for colorectal cancer. Br. J. Surg. 2004; 91(5): $1111-24$

\section{Source of Support: Nil; Conflict of Interest: None}

Submitted: 18-04-2019; Accepted: 13-05-2019; Published online: 30-06-2019 